\title{
ETHICAL CONSIDERATIONS IN MEDICAL PRACTICE AND RESEARCH
}

\author{
Rajeev Kumar' ${ }^{1}$ Leena Kumari², Vijay Kumar ${ }^{3}$ \\ ${ }^{1}$ HOD, Department of FMT, SGT University, Gurgaon, Haryana. \\ ${ }_{2}^{2}$ MSc. Forensic Science Student, SGT University, Gurgaon, Haryana. \\ ${ }^{3} \mathrm{HOD}$, Department of Audiology, Amity University Gurgaon, Haryana.
}

\begin{abstract}
Ethics is governed by moral principles that define human conduct for an individual, group or society. Role of ethics has become extremely important in medical practice and research. Medical practice or any scientific act related to human participation in research should be carefully calibrated safeguarding the ethical interest of the patient/participant. The philosophy of medical intervention should be to integrate a transparent health care system that focuses on consistent delivery of evidence based care at the right time in the right environment and in right manner. In futuristic healthcare sector, upcoming professionals should be adequately sensitized to protect and respect the ethical interest of the patients.
\end{abstract}

\section{KEYWORDS}

Ethics, Healthcare, Research.

HOW TO CITE THIS ARTICLE: Kumar R, Kumari L, Kumar V. Ethical considerations in medical practice and research. J. Evolution Med. Dent. Sci. 2016;5(7):343-345, DOI: 10.14260/jemds/2016/75

\section{INTRODUCTION}

Ethics is the branch of philosophy centered on the study of the moral principles that define a standard of human conduct for an individual, group or culture (Snyder E. J. and Gauthier C. C., 2008). ${ }^{1}$ Whereas Medical Ethics (ME) is the study of how the practice of medicine correlates with acceptable conduct is specifically termed as Medical Ethics. The ME is defined as code of behaviour accepted voluntarily within the profession. The ME also enables us to understand the dynamics between ethics and law of the land.

Ethics may differ marginally to significantly among different cultures, population, socioeconomic, health status, disability condition and profession. Therefore, knowledge of ME is extremely important to execute routine diagnostic and intervention practice. The ME needs to be incorporated and designed in medical training and practice to address the evergrowing dynamic challenges. The role of it in today's culturally diversified health care environments to facilitate decision making model to solve frequently cropping ethical dilemmas. It also helps in addressing ethical challenges and identifying the ethical duties and actions of health care professionals.

\section{Historical Preview of Medical Ethics}

First evidence of medical ethics dates back in the Oath of Hippocrates, which was likely to be written in $5^{\text {th }}$ century B.C. Subsequently, the Code of Conduct for Physicians was introduced. It has been modified over the time and since early eighteen century it has been used in various forms at many medical school commencement ceremonies. Nearly twenty four century after the original oath of Hippocrates, the first code of ethics was approved in 1847 at the first meeting of American Medical Association known as American Medical Association (AMA) Code of Ethics. ${ }^{2}$

Financial or Other, Competing Interest: None.

Submission 10-11-2015, Peer Review 11-11-2015,

Acceptance 16-01-2016, Published 25-01-2016.

Corresponding Author:

Dr. Leena Kumari,

MSc. Forensic Science Student,

SGT University, Gurgaon, Haryana.

E-mail: leena.bhardwaj2000@gmail.com

DOI: $10.14260 /$ jemds $/ 2016 / 75$
It emphasizes the rules of professional behaviour and also clarifies the training requirement for participating physicians. Advancement of medical technology as per the socio-cultural framework of the country, periodically the code of ethics has undergone several revisions.

Exactly one century after the AMA code, Nuremberg Code. ${ }^{3}$ was developed. It was the outcome of the indictment and prosecution of Nazi physician's accusing of human participates in experimentation. It was the landmark document for promoting human rights protection in research. The concept of informed voluntary consent came forth with this document. Several historical events provided momentum to the evolution of medical ethics. The Tuskegee syphilis study has marred the reputation of healthcare irremediably. In this U.S. Public Health Service study, spanning 40 years from 1932 to 1972, 399 African-American males from rural Alabama were given non-curative antibiotic courses for syphilis.

The reported purpose of the study was infection control, but with the expert's opinion the study aimed not only to control disease transmission, but also to document the progression of disease through its stages. Despite objectives to ethical premises of this investigation as early 1965, the study continued for another seven years before it was eventually declared unethical by a nine-member advisory panel of the Department of Health, Education and Welfare. It has immense negative societal impact. In the words of Dr. Vanessa Northington Gamble, "the Tuskegee study has come to symbolize racism in medicine, misconduct in human research, the arrogance of physicians and government abuse of black people."

Concern for the protection of human research subjects leads to formation of National Commission for the Protection of Human Subjects. ${ }^{4}$ in 1974 and subsequent publications was referred as The Belmont Report.5; Ethical Principles and Guidelines for the protection of human subjects of research. The Belmont Report lead to the requirement that all research institutions receiving federal funding must form an Institutional Review Board (IRB). Members of IRB shall generally include both researchers and lay persons. IRB review both proposals for research protocols as well as the 
means by which informed consent is obtained for study projects.

The Medical Ethics is based on a series of ethical principles that are relevant to medical practice and patient care. In 1979, Beauchamp, Tom and James. ${ }^{6}$ penned the significant work which was later recognized as Principles of Biomedical Ethics. The four basic ethical principles essential to medicine were - a) Autonomy b) Beneficence c) NonMalfeasance and d) Distributive Justice. This principle was first developed by Tom Beauchamp and James Childress in 1979 in their book, Principles of Biomedical Ethics. The Principle of Respect for Autonomy highlights capable patients must be allowed to accept or refuse recommended medical treatment. Autonomy is defined as the capacity of selfdetermination or the capacity involves to make own decision. The Principle of Beneficence states that medical practitioner should act in the best interest of the patient. The relevant harm like pain, suffering, disease, disability and death should be prevented during delivery of healthcare. The Principle of NonMalfeasance ensures that medical practitioner must not harm the patient.

Along with this principle, act in best interest of patient becomes complicated. Medical interventions normally involve risks and benefits. It means that the Principle of Beneficence and the Principle of Non-Malfeasance need to be applied together. For instance before prescribing a medicine a practitioner must weigh the expected beneficial effects with the potential harmful side effects. Contextually this capacity involves ability to make and communicate healthcare decisions. Voluntary Informed Consent is required to implement this principle. Controversy arises here over the determination that is unable to make decisions like young people below the legal age of consent, i.e. 18 years, patient suffering from early and middle stages of Alzheimer's disease.

The Principle of Distributive Justice: Healthcare resources should be distributed in a fair way among the members of society. The Principle of Distributive Justice applies most readily on the governmental and Institutional level, in determining how much of our tax money will go to health care and in deciding how an institutions resources will be allocated. Sometimes practitioners faces "ethical dilemma" in which the force of the conflicting principles seems to be equal. In these cases, it may be helpful to consult with hospital ethics committee. This committee will consider the interest of everyone involved in the situation, as well as relevant ethical principles, and may be able to recommend courses of action aimed at resolving the conflict.

\section{Advance Directive}

It is term used to refer to any document that makes the wishes of a capable patient clear for a time in the future when the patient is no longer able to make or communicate health care decisions. Together the Living Will and the document used to appoint a healthcare agent the power of attorney for health care is known Advance Directive. In 1976, California passed the Natural Death Act. ${ }^{7}$ and became the first state to offer its citizens a way to make their wishes for the end-of-life care known in advance.

The Living Will expresses a patient's wishes that medical technology not be used to prolong the dying process. It is legally executed document authorizing physicians to withhold or withdraw life sustaining medical treatment when the patient has a terminal condition or permanently unconscious as in persistent vegetative state.

It is supported by the Principle of Respect of Autonomy. ${ }^{7}$ The Power of Attorney for Healthcare is a legal document that a capable patient uses to appoint a health surrogate or proxy; to make medical decisions when a patient is no longer able to communicate such decisions. The Power of Attorney of Healthcare may also include statement of specific desires for treatment at the end of life. This second type of Advance Directive is supported by the Principle of Respect for Dignity and the principle of Respect for Autonomy. When the patients have not executed a Living Will or Power of Attorney for Healthcare, Common Law and State Statute Permit Decisions to be made by family members. In most states the hierarchy for decision making, without a power of Attorney for health is considered illegal.

\section{ME: Indian Perspective}

In Indian context, Indian Council of Medical Research (ICMR) proposed guidelines for Biomedical research. ${ }^{8}$ (1980). Policy Statement on Ethical Considerations involved in research on Human Subjects (2015) pending for approval in Parliament. Some of the key features are essentiality of research, voluntariness of participation, informed consent, onexploitation, vulnerable populations, privacy and confidentiality, risk minimization, professional competence of researcher accountability and transparency maximization of public interest and distributive justice institutional arrangements public domain totality of responsibility.

\section{Futuristic Healthcare Delivery Model}

Focused on the patient as a consumer, customer relationship management technology has potential to generate and manage the emerging ethical demand. Integrated and transparent health care system that focuses on consistent delivery of evidence based care at the right time in the right environment. Sustainable medical systems has to operate as well orchestrated system of healthcare management to ensure quality based and efficient health care economy to support and sustain different types of risk and quality based care models.

In the contest of fast moving global digital village and constantly changing technology have some disadvantages in spite of its fruitfulness. Bioterrorism due to Nanotechnology in the widest futuristic scenario, tiny nanorobots in our blood stream could detect diseases are few examples. After a few decades they might even eradicate the word symptom in as much as no one would have symptoms any more. This microscopic robot would send alerts to our smart phones or digital contact lenses before disease could develop in our body. If it becomes reality and microrobots swimming in body fluids are already out there, how can we prevent terrorists from trying to hack these devices controlling not only our health but our lives.

Hacking medical devices it has already been proven that pacemakers and insulin pumps can be hacked. Security experts have warned us that someone would be murdered through these methods anytime soon. How can we prevent wearable devices that are connected to our physiological system from being hacked and controlled from distance? Transhumanism and Singularitarianism Transhumanism (Abbreviated as $\mathrm{H}+$ ) is an international and intellectual movement that aims to transform the human conditions by 
developing and creating widely available technologies to greatly enhance human intellectual, physical and psychological capacities. Fundamental ideas of Transhumanism were first advanced in 1923 by British Geneticist J.B.S. Haldane in his essay Science and Future, which predicted that great benefits would come from applications of advanced sciences to human biology.

Singularitarianism is a movement defined by the belief that a technological singularity the creation of superintelligence will likely to happen in medium future and that deliberate action ought to be taken to ensure that significantly benefits humans. We should be skeptical and analytical before accepting major philosophies about the future. Scanning ourselves at home now physicians are worried because patients scan themselves, do blood test and even genetic analysis at home? Will us able to persuade such patients to turn to doctors.

Therefore, every healthcare professional should be sensitized about the ethical rights of the clients and one need to practice by maintaining benchmark of professional cum ethical practice.

\section{REFERENCES}

1. Snyder EJ and Gauthier CC (2008). Evidence based medical ethics: Case for practice-based learning. Humana press.

2. American Medical Association. Principles of medical ethics. Appendix F. 1957:355-257. In: Baker RB. The American Medical Ethics Revolution. Baltimore, MD: John Hopkins University Press.
3. The Nuremberg Code [from Trials of War Criminals before the Nuremberg Military Tribunals under Control Council Law No. 10. Nuremberg, October 1946-April 1949. Washington, D.C.: U.S. G.P.O, 1949-1953.]

4. World Medical Association. Declaration of Helsinki. Ethical principles for medical research involving human subjects [Internet]. Seoul:WMA 2008 [cited 2012 May 31]. Available from: http://www.wma.net/en/30publications/10policies/b $3 /$

5. The Belmont Report: Ethical Principles and Guidelines for the Protection of Human Subjects of Research (The National Commission for the Protection of Human Subjects of Biomedical and Behavioural Research, 1979) available from http://ohsr.od.nih.gov/mpa/belmont.php3, INTERNET, p.3.

6. Tom L Beauchamp and James F Childress. 1979. Principles of biomedical ethics. New York: Oxford University Press.

7. World Medical Association International Code of Medical Ethics as amended by the $35^{\text {th }}$ World Medical Assembly, Venice, Italy, October 1983.

8. Indian Council of Medical Research. Ethical guidelines for biomedical research on human participants [Internet]. New Delhi: Indian Council of Medical Research; 2008 Available from: http://icmr.nic.in/ethical_guidelines.pdf 